\title{
Localization of Interacting Fermions in the Aubry-André Model
}

\author{
Vieri Mastropietro \\ Università degli Studi di Milano, Via Saldini, 50, 20133 Milano, Italy
}

(Received 8 September 2015; published 26 October 2015)

\begin{abstract}
We consider interacting electrons in a one-dimensional lattice with an incommensurate Aubry-André potential in the regime when the single-particle eigenstates are localized. We rigorously establish the persistence of ground state localization in the presence of weak many-body interaction, for almost all the chemical potentials. The proof uses a quantum many-body extension of methods adopted for the stability of tori of nearly integrable Hamiltonian systems and relies on number-theoretic properties of the potential incommensurate frequency.
\end{abstract}

Interacting fermions with a quasiperiodic potential describe quasicrystals or crystals with incommensurate charge density waves and have a number of features in common with systems with random disorder; in particular, Anderson localization [1] can occur in the single-particle eigenstates. The paradigmatic model for such systems is the interacting Aubry-André model [2], which can be equivalently written as the Heisenberg $X X Z$ spin chain with an incommensurate magnetic field. The interest of this model relies from one side on the fact that, despite its simplicity, it has a number of nontrivial features common to more realistic systems, like a metal-insulator transition even at the single-particle level; on the other, it provides an accurate description of cold atoms with quasirandom optical lattices generated by two or more incommensurate frequencies [3-5]. At the single-particle level, that is neglecting the many-body interaction, its properties follow from the Harper or almost-Mathieu equation, which has been extensively studied in the last 30 years; it is known, in particular, that the spectrum is a Cantor set [6] with a dense set of gaps and that its eigenfunctions are extended [7] or localized [8] varying the strength of the potential. Such remarkable properties are related to a deep connection between the noninteracting Aubry-André model and the Kolmogorov-Arnold-Moser theorem expressing the stability of invariant tori in quasi-integrable Hamiltonian systems.

Much less is known when a many-body interaction is present, and one of the key questions is which is the fate of Anderson localization, which is a single-particle phenomenon. Such a problem is of general interest in disordered quantum matter (a quasiperiodic potential can be considered a quasirandom disorder). In the case of truly random disorder, localization in the presence of interaction is supported by analytical arguments $[9,10]$ and rigorous proofs [11] (requiring, however, an unproven assumption), and important consequences of such a property for nonequilibrium dynamics have been exploited [12-16]. A complete proof of localization in the presence of interaction, even if limited to the ground state, is, however, still lacking in the random case. In the interacting AubryAndré model, analytical results have been established $[17,18]$ only in the extended regime; in the localized phase numerical simulations [19] and cold-atom experiments [5] support the stability of localization for attractive or repulsive interactions.

In this Letter we rigorously establish in the Aubry-André model the persistence of ground state localization in presence of weak many-body interaction, for almost all values of the chemical potentials. The analysis relies heavily on number-theoretical properties of the frequency of the incommensurate potential, which play a key role already in the noninteracting case; however, the structure of small divisors is made involved by the presence of loops caused by the many-body interaction. Our result is obtained by a new technique based on a combination of multiscale methods developed for the classical Lindstedt series for Kolmogorov-Arnold-Moser invariant tori in nearly integrable Hamiltonian systems [20] combined with nonperturbative renormalization group methods [21].

The Hamiltonian of the interacting Aubry-André model is

$$
\begin{aligned}
H= & -\varepsilon \sum_{x}\left(a_{x+1}^{+} a_{x}+a_{x}^{+} a_{x-1}^{-}\right)+\mu \sum_{x} a_{x}^{+} a_{x}^{-} \\
& +u \sum_{x} \phi_{x} a_{x}^{+} a_{x}^{-}+U \sum_{x} a_{x}^{+} a_{x}^{-} a_{x+1}^{-} a_{x+1}^{+},
\end{aligned}
$$

with $a_{x}^{ \pm}$fermionic creation or annihilation operators, $x$ points on a one-dimensional lattice with step 1 and Dirichlet boundary conditions, and $\phi_{x}$ a potential incommensurate with the lattice $\phi_{x}=\cos (2 \pi \omega x)$ and $\omega$ irrational; it is convenient, as in the analysis of the Harper equation, to assume that $\omega$ verifies a Diophantine condition (valid on a full measure set)

$$
\|x \omega\| \geq C|x|^{-\tau}, \quad x \neq 0,
$$


with the double vertical bars the norm on the one-dimensional torus of period 1. For definiteness we are choosing coordinates so that $\phi_{x}$ is even with respect to $x=0$.

Ground state localization implies zero temperature exponential decay for large values of the coordinate difference in the thermodynamical correlations, for instance, in the two-point function $\left\langle T a_{\mathbf{x}}^{-} a_{\mathbf{y}}^{+}\right\rangle$, where $T$ is the time order product, $a_{\mathbf{x}}^{ \pm}=e^{H x_{0}} a_{x}^{ \pm} e^{-H x_{0}}, \mathbf{x}=\left(x_{0}, x\right)$, and \langle\rangle$=\left(\operatorname{Tr} e^{-\beta H} \cdot / \operatorname{Tr} e^{-\beta H}\right)$. In the absence of hopping or interaction $U=\varepsilon=0$ (molecular limit) there is perfect localization and, if $\left.\left\langle T a_{\mathbf{x}}^{-} a_{\mathbf{y}}^{+}\right\rangle\right|_{U=\varepsilon=0}=g(\mathbf{x}, \mathbf{y})$,

$$
g(\mathbf{x}, \mathbf{y})=\delta_{x, y} \int d k_{0} \frac{e^{i k_{0}\left(x_{0}-y_{0}\right)}}{-i k_{0}+u \phi_{x}-\mu},
$$

which is exactly vanishing for fermions with different coordinates. We can now consider small $U, \varepsilon$ (compared to $u$ ) and see if the theory is analytically close to the molecular limit. The interacting two-point function can be written in terms of a perturbative expansion in $U, \varepsilon$ and each term of the series is expressed in terms of Feynman graphs given by sums of products of propagators (3), whose singularities naturally determine the physical properties.

As the frequency $\omega$ is irrational, $(\omega x)_{\bmod 1}$ fills densely the interval $(-1 / 2,1 / 2]$ so that the denominator $u \phi_{x}-\mu$ can be arbitrarily small; this happens when $(\omega x)_{\bmod 1}$ is close to $\pm(\omega \bar{x})_{\bmod 1}$ with $\mu=u \cos 2 \pi \omega \bar{x}$; then for small $\left(\omega x^{\prime}\right)_{\bmod 1}$ one has $\phi_{x^{\prime} \pm \bar{x}}-\mu \sim \pm\left(\omega x^{\prime}\right)_{\bmod 1}$. Note the close analogy between the two-point function (3) at the molecular limit $u=\varepsilon=0$, with the propagator in the free Fermi gas limit $U=u=0$, namely, $\left(-i k_{0}+\varepsilon \cos k-\mu\right)^{-1}$; the points $\pm \bar{x}$ have the same role as the Fermi momenta $\pm p_{F}$ such that $\mu=\cos p_{F}$, so it is natural to call them Fermi coordinates. This relation between molecular and free gas limit is known as Aubry duality.

We expect that the interaction causes a renormalization of the chemical potential and it is then convenient to fix the interacting chemical potential by choosing properly its bare value; in particular, the bare chemical potential is taken as $\mu=u \cos (2 \pi \omega \bar{x})+\varepsilon \nu$, and $\nu$ is determined by requiring the dressed chemical potential to be $u \cos (2 \pi \omega \bar{x})$. There are two main cases to be considered, corresponding to a choice of the chemical potential in one of the gaps of the noninteracting spectrum (which are a dense set) or outside a gap. The gapped case is when fermions living close to different Fermi points, that is, with coordinate $x^{\prime}-\bar{x}$ and $x^{\prime}+\bar{x}$ with $\left(\omega x^{\prime}\right)_{\bmod 1}$ small, are exactly connected by the hopping or by the interaction, which causes jumps of $0, \pm 1$ sites; this happens when $2 \bar{x}$ is an integer and causes the gaps in the noninteracting spectrum, exactly like a periodic potential produces gaps in the free Fermi gas limit (in space of momenta instead of coordinates). In the second case we choose the chemical potentials outside the gaps, and we do that by requiring a Diophantine condition for the Fermi coordinates, excluding only a zero measure set of values

$$
\|\omega x \pm 2 \omega \bar{x}\| \geq C|x|^{-\tau}, \quad x \neq 0 .
$$

In this case, fermions with coordinates $x^{\prime}-\bar{x}$ and $x^{\prime}+\bar{x}$ with $\left(\omega x^{\prime}\right)_{\bmod 1}$ small cannot be exactly connected by the hopping or by the interaction (but arbitrarily closely connected). Note that $\left|\hat{g}\left(k_{0}, x\right)\right| \leq C|x|^{\tau}$; that is, the denominator can be very small for large $x$. This is a sort of ultraviolet-infrared mixing, a phenomenon typical of incommensurate potentials. Our main result is the following.

Theorem 1.-Let us fix $u=1, U=\lambda \varepsilon$, and assume $\varepsilon, \lambda$ small and $\omega$ verifying (2), $\mu=\phi_{\bar{x}}+\varepsilon \nu$. Then if $\bar{x}$ verifies (4) (chemical potentials outside the gaps of the noninteracting spectrum) or if $\bar{x}$ is a half integer (chemical potentials in the gaps of the noninteracting spectrum), then, for a suitable choice of $\nu$, the zero temperature two-point function obeys to

$$
\left|\left\langle T a_{\mathbf{x}}^{-} a_{\mathbf{y}}^{+}\right\rangle\right| \leq C e^{-\xi|x-y|} F\left(x_{0}-y_{0}\right),
$$

with $\xi=C|\log | \varepsilon \|, \quad C \quad$ a constant, and $F=[1 / 1+$ $\left.\left.\left(\Delta \mid x_{0}-y_{0}\right) \mid\right)^{N}\right]$ with $\Delta=[1+\min (|x|,|y|)]^{-\tau}$ if $\bar{x}$ verifies (4), while $\Delta=a|\varepsilon|^{2 \bar{x}}+O\left(\varepsilon^{2 \bar{x}+1}\right)$ and $a>0$ if $\bar{x}$ is a half integer.

The above result establishes exponential decay of the two-point function, implying Anderson localization for the ground states of the interacting system, both when the chemical potentials are in the center of the gaps or outside the gaps; in the first case there is a fast decay in time with rate proportional to the gap size.

Proof of Theorem 1.-We present here the key steps of the proof (more technical details will be published elsewhere [22]). The two-point function is obtained by the second derivative of the generating function

$$
e^{W(\phi)}=\int P(d \psi) e^{V(\psi)+(\psi, \phi)},
$$

with

$$
\begin{aligned}
V= & -\lambda \int d \mathbf{x} \psi_{\mathbf{x}}^{+} \psi_{\mathbf{x}}^{-} \psi_{\mathbf{x}+\mathbf{e}_{1}}^{+} \psi_{\mathbf{x}+\mathbf{e}_{1}}^{-} \\
& +\varepsilon \int d \mathbf{x}\left(\psi_{\mathbf{x}}^{+} \psi_{\mathbf{x}+e_{1}}^{-}+\psi_{\mathbf{x}+e_{1}}^{+} \psi_{\mathbf{x}}^{+}\right)+\nu \int d \mathbf{x} \psi_{\mathbf{x}}^{+} \psi_{\mathbf{x}}^{-},
\end{aligned}
$$

where $\psi$ are Grassmann variables, $\phi$ is the external source, $\int d \mathbf{x}=\int d x_{0} \sum_{x}, \mathbf{e}_{1}=(0,1)$, and $P(d \psi)$ is the fermionic integration with propagator (3). We introduce a cutoff smooth function $\chi_{\rho}\left(k_{0}, x\right)$ which is nonvanishing for $\sqrt{k_{0}^{2}+\left\{\left[\omega(x-\rho \bar{x})_{\bmod 1}\right]^{2}\right\}} \leq \gamma$, where $\rho= \pm 1$ and $\gamma>1$ is a suitable constant (to be fixed below); therefore, we can write the propagator as

$$
\hat{g}\left(k_{0}, x\right)=\hat{g}^{(u \cdot v \cdot)}\left(k_{0}, x\right)+\sum_{\rho= \pm} \hat{g}_{\rho}\left(k_{0}, x\right),
$$


where $\hat{g}_{\rho}\left(k_{0}, x\right)=\left[\chi_{\rho}\left(k_{0}, x\right) /-i k_{0}+\phi_{x}-\mu\right]$, and correspondingly $\psi_{k_{0}, x}=\psi_{k_{0}, x}^{(u . v)}+\sum_{\rho= \pm 1} \psi_{\rho, k_{0}, x}$. This simply says that we are rewriting the fermionic field as sum of two independent fields living close to one of the Fermi points, up to a regular field. We can further decompose

$$
\hat{g}_{\rho}\left(k_{0}, x\right)=\sum_{h=-\infty}^{0} \hat{g}_{\rho}^{(h)}\left(k_{0}, x\right)
$$

with $\hat{g}_{\rho}^{(h)}\left(k_{0}, x\right)$ similar to $\hat{g}_{\rho}^{(h)}\left(k_{0}, x\right)$ with $\chi$ replaced by $f_{h}$ where $f_{h}\left(k_{0}, x^{\prime}\right)$ is nonvanishing in a region $\sqrt{k_{0}^{2}+\left[\left(\omega x^{\prime}\right)_{\bmod 1}\right]^{2}} \sim \gamma^{h}$. After the integration of $\psi^{(u \cdot v .)}, \psi^{(0)}, \ldots, \psi^{(h+1)}$ the generating function has the form

$$
e^{W(\phi)}=\int P\left(d \psi^{\leq h}\right) e^{V^{(h)}(\psi)+B^{(h)}(\psi, \phi)},
$$

where $P\left(d \psi^{\leq h}\right)$ has propagator $g_{\rho}^{(\leq h)}=\sum_{k=-\infty}^{h} g_{\rho}^{(k)}$ and $V^{(h)}(\psi)$ is given by

$$
V^{(h)}(\psi)=\sum_{m} W_{m}^{(h)} \psi_{\rho_{1}, x_{0,1}, x_{1}^{\prime}+\rho_{1} \bar{x}}^{\varepsilon_{1}(h)} \cdots \psi_{\rho_{m}, x_{0, m}, x_{m}^{\prime}+\rho_{m} \bar{x}}^{\varepsilon_{m}(\leq h)}
$$

and the kernels $W_{m}^{(h)}$ are the sum of Feynman diagrams obtained connecting vertices $\varepsilon, \lambda$, or $\nu$ with propagators $g^{(k)}$ with $k>h ; B^{(h)}$ is given by a similar expression with the only difference that some of the external lines are associated with $\phi$ fields. In each of the Feynman diagrams contributing to $W_{m}^{(h)}$ there is a tree of propagators connecting all the external lines and the vertices; the coordinates $x_{i}, x_{j}$ of two external lines are such that

$$
x_{i}-x_{j}=x_{i}^{\prime}+\rho_{i} \bar{\omega}-x_{j}^{\prime}-\rho_{j} \bar{\omega}=\sum_{\alpha}^{*} \delta_{\alpha},
$$

where the sum is over the vertices in the path of the tree connecting $i$ and $j$ and $\delta_{\alpha}=(0,1,-1)$ (see Fig. 1) is associated with the line connected to the vertex $\alpha$. Of course

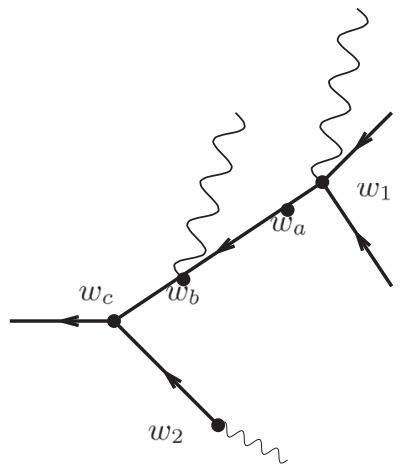

FIG. 1. An example of a tree; the solid lines represent propagators and the wiggly lines the external lines $x_{w_{1}}-x_{w_{2}}=$ $\delta_{1}+\delta_{a}+\delta_{b}+\delta_{c}+\delta_{2}$. $x_{i}-x_{j}$ has an integer value. Each kernel $W_{m}^{(h)}$ is expressed by a power series in $\varepsilon, \nu, \lambda$, and one has to show convergence uniformly in the renormalization group step $|h|$; however, standard power counting arguments says that the theory is nonrenormalizable, as the naive degree of divergence is $k-1$ if $k$ is the order of the graph. Power counting is not sufficient and one has to use a more accurate analysis exploiting the number-theoretic properties of the frequency.

In order to do that it is convenient, given a Feynman graph, to consider a maximally connected subset of lines corresponding to propagators with scale $h \geq h_{v}$ with at least a scale $h_{v}$, and we call it cluster $v$; the external lines have scale smaller then $h_{v}$. Therefore, each Feynman graph is associated with a hierarchy of clusters; inside each cluster $v$ there are $S_{v}$ maximal clusters, that is, clusters contained only in the cluster $v$ and not in any smaller one, or trivial clusters given by a single vertex. Each of such $S_{v}$ clusters are connected by a tree of propagators with scale $h_{v}$; by integrating the propagators over the time, and using that $\int d x_{0}\left|g_{\rho}^{(h)}\left(x_{0}, x\right)\right| \leq C \gamma^{-h}$ and that $\left|g_{\rho}^{(h)}\left(x_{0}, x\right)\right| \leq C$, we get that each graph of order $n$ contributing to $W_{m}^{(h)}$ is bounded by

$$
C^{n} \mathcal{E}^{n} \prod_{v} \gamma^{-h_{v}\left(S_{v}-1\right)},
$$

where $v$ are the clusters and $h_{v} \leq 0$. As $S_{v} \geq 1$ the above estimate says that the size of the graph increases with $|h|$ and diverges in the zero temperature limit. In getting (12), we have, however, not used a crucial property implied by the Diophantine condition: namely, that if the denominators associated with the external lines have the same size, the difference of coordinates must be or $0, \pm 2 \bar{x}$ (the second case only for $\bar{x}$ half integer) or very large; in this second case there is a large number of hopping or interactions terms by (11) and therefore a decaying factor associated with a high power of $\varepsilon$. This suggests to distinguish in $V^{(h)}$ two kinds of terms, the resonant terms, such that the coordinates $x_{i}^{\prime}$ measured from the Fermi coordinates of the external fields are all equal, and the remaining nonresonant terms. We define, as usual in the theory of renormalization, a localization operator $\mathcal{L}$ acting only on the resonant terms and setting all the temporal coordinates of the external fields equal. We split the resonant terms in a local part, where $\mathcal{L}$ applies, and a renormalized part, where $\mathcal{R}=$ $1-\mathcal{L}$ applies. In the $\mathcal{R}$ part there is at least a difference of fields $\left(\psi_{\rho, x_{0, i}, x^{\prime}+\rho \bar{x}}^{\varepsilon(\leq h)}-\psi_{\rho, x_{0, i}, x^{\prime}+\rho \bar{x}}^{\varepsilon(\leq h)}\right)$ and this produces a extra gain $\gamma^{h^{\prime}{ }^{\prime} h_{v}}$, if $v$ is a resonant cluster and $v^{\prime}$ is the minimal cluster containing it. Regarding the local terms, they are proportional to a monomial of fields with the same coordinates $\mathbf{x}^{\prime}$; hence, anticommutativity implies that terms with more than four fields are exactly vanishing. The possibly nonvanishing quartic terms have the form 


$$
\varepsilon \lambda_{h} \int d \mathbf{x} \psi_{+, x_{0}, x^{\prime}+\bar{x}}^{+} \psi_{+, x_{0}, x^{\prime}+\bar{x}}^{-} \psi_{-, x_{0}, x^{\prime}-\bar{x}}^{+} \psi_{-, x_{0}, x^{\prime}-\bar{x}}^{-} .
$$

When $\bar{x}$ verifies (4) (and hence cannot be an half-integer) such terms are vanishing as there are fields with coordinate difference $2 \bar{x}$ which by (11) should be an integer. When $\bar{x}$ is a half integer local quartic terms exist and in such a case $\lambda_{h}=O\left(\varepsilon^{2 \bar{x}-1} \lambda\right)$. Similarly the quadratic terms are of two kinds: one is $\varepsilon \nu_{h} \sum_{\rho} \int d \mathbf{x} \psi_{+, x_{0}, x^{\prime}+\rho \bar{x}}^{+} \psi_{+, x_{0}, x^{\prime}+\rho \bar{x}}^{-}$representing the renormalization of the chemical potential; the other is $\sigma_{h} \sum_{\rho} \int d \mathbf{x} \psi_{+, x_{0}, x^{\prime}+\rho \bar{x}}^{+} \psi_{+, x_{0}, x^{\prime}-\rho \bar{x}}^{-}$and is vanishing when $\bar{x}$ verifies (4) while it is present when $\bar{x}$ is a half integer and in such a case $\sigma_{h}=O\left(\varepsilon^{2 \bar{x}}\right)$.

We have now to consider the contributions from the nonresonant terms of the effective potential $V^{(h)}$. In such terms there are least two external fields with coordinate $x_{1}^{\prime}$, $x_{2}^{\prime}$ with $x_{1}^{\prime} \neq x_{2}^{\prime}$; if $|h|$ is large the corresponding divisors are small $O\left(\gamma^{h}\right)$ but the coordinate difference is large. More quantitatively, if $m=\sum_{\alpha}^{*} \delta_{\alpha} \neq 0$, see (11) and $v^{\prime}$ is the cluster containing $v$

$$
\begin{aligned}
2 \gamma^{h_{v^{\prime}}} & \geq\left\|\left(\omega x_{1}^{\prime}\right)\right\|+\left\|\left(\omega x_{2}^{\prime}\right)\right\| \geq\left\|\omega\left(x_{1}^{\prime}-x_{2}^{\prime}\right)\right\| \\
& =\left\|\omega\left(\rho_{2}-\rho_{1}\right) \bar{x}+m \omega\right\| \geq C_{0}|\delta 2 \bar{x}+| m \|^{-\tau},
\end{aligned}
$$

with $\delta=0$ when $\bar{x}$ verifies (4) and $\delta=1$ when it is a half integer; the last inequality follows from (2) and (4). Therefore, $|m| \geq \tilde{C} \gamma^{-h / \tau}$; that is, $m$ must be very large if the divisors are small and not coinciding. In a nonresonant cluster there then are necessarily a large number of vertices $N$ so that $N \geq|m| \geq \tilde{C} \gamma^{-h / \tau}$; by taking into account the hierarchy of clusters one gets

$$
\varepsilon^{n} \leq \varepsilon^{n / 2} \prod_{v} \varepsilon^{C 2^{h_{v}} \gamma^{-h_{v} / \tau} S_{v}^{\mathrm{NR}}},
$$

where $S_{v}^{\mathrm{NR}}$ are the nonresonant clusters contained in $v$; that is, a decay factor is associated with each nonresonant cluster. The power counting bound (12), apparently saying that the theory is nonrenormalizable, is therefore replaced by

$$
G \varepsilon^{n / 2}\left[\prod_{v} \gamma^{-h_{v}\left(S_{v}-1\right)}\right]\left[\prod_{v} \varepsilon^{C 2^{h_{v}} \gamma^{-h_{v} / \tau} S_{v}^{\mathrm{NR}}}\right]\left[\prod_{v}^{*} \gamma^{h_{v^{\prime}}-h_{v}}\right],
$$

where $\prod_{v}^{*}$ is over the resonant terms and $G$ contains the product of running coupling constants. Note that $S_{v}=S_{v}^{\mathrm{R}}+S_{v}^{\mathrm{NR}}+S_{v}^{e}$, where $S_{v}^{\mathrm{R}}$ is the number of resonant clusters and $S_{v}^{e}$ is the number of end points with scale $h_{v}$ in the cluster $v$. The above bound is true not only for the single graph, but for the sum of all graphs contributing to order $n$ and summing over all the possible choices of external lines of the clusters. Note that $\left[\prod_{v} \gamma^{-h_{v}\left(S_{v}^{\mathrm{R}}-1\right)}\right]\left[\prod_{v}^{*} \gamma^{h_{v^{\prime}}-h_{v}}\right] \leq 1$.
Finally one has to discuss the flow of the effective running coupling constant. When $\bar{x}$ verifies (4) the only running coupling constant is $\nu_{h}$, expressing the renormalization of the chemical potential, and by choosing properly $\nu$ then by a fixed point argument $\nu_{h}=O\left(\gamma^{h}\right)$. When $\bar{x}$ is a half integer then one has also a mass term $\sigma_{h}=a \varepsilon^{2 \bar{x}}+\left(\varepsilon^{2 \bar{x}+1}\right)$ and this implies that there is a mass scale $h^{*}$ of the order of the log of the mass. The effective coupling is $\lambda_{h}=\lambda_{0}\left[1+O\left(\left|h^{*}\right| \varepsilon\right)\right]$ and as $\lambda_{0}=O\left(\varepsilon^{2 \bar{x}-1} \lambda\right)$ then $\gamma^{-h}\left|\varepsilon \lambda_{h}\right|$ is small. From (16) we have finally that the size of the $n$th contribution to $W_{m}^{(h)}$ is

$$
\varepsilon^{n / 2}\left[\prod_{v}\left(\gamma^{-h_{v}} \varepsilon^{C 2^{h_{v}} \gamma^{-h_{v} / \tau}}\right)^{S_{v}^{\mathrm{NR}}}\right] \leq C^{n} \varepsilon^{n / 2}\left[\prod_{v} \gamma^{h_{v} S_{v}^{\mathrm{NR}}}\right] .
$$

If $\gamma^{1 / \tau}>2$ and $\varepsilon$ is small one can then sum over $\left\{h_{v}\right\}$ and get an $\varepsilon^{n / 2} C^{n}$ bound. When $S_{v}^{\mathrm{NR}} \neq 0$ this is trivial. If $S_{v}^{\mathrm{NR}}=$ 0 then $v$ is resonant and (a) when $\bar{x}$ is a half integer one uses the gap saying that the number of scales is $O(\log \varepsilon)$, and (b) when $\bar{x}$ verifies (4), if $v$ has two external lines then there is no sum as $h_{v}=h_{v^{\prime}}+1$ by the support properties of $f_{h}$ as the internal lines of $v$ have the same $k_{0}, x^{\prime}$ as the external ones; if it has more than four lines, $v$ is resonant and the external lines have the same $\rho$ index by (11) [see after (13)]; therefore, there are at least two couples of identical fields $\psi_{\rho, x^{\prime}+\rho \bar{x}, x_{0, i}}^{\varepsilon} \psi_{\rho, x^{\prime}+\rho \bar{x}, x_{0, j}}^{\varepsilon}=\psi_{\rho, x^{\prime}+\rho \bar{x}, x_{0, i}}^{\varepsilon}\left(\psi_{\rho, x^{\prime}+\rho \bar{x}, x_{0, j}}^{\varepsilon}-\psi_{\rho, x^{\prime}+\rho \bar{x}, x_{0, i}}^{\varepsilon}\right)$ so that there is at least an extra factor $\gamma^{h_{v^{\prime}}-h_{v}}$ with respect to the one already used in (16), allowing the sum over $h_{v}$. A similar analysis can be done for the terms with two external lines $\phi$ contributing to the two-point function; the exponential decay in the coordinate follows again from (11) as the only nonvanishing contributions have an order greater than $|x-y| / 2$.

In conclusion we have rigorously established the persistence of localization of the ground state for almost all the chemical potentials and in the presence of attractive or repulsive weak interaction. The analysis relies heavily on the number-theoretic properties of the frequency of the quasiperiodic potential. Our result would imply localization of all the eigenfunctions in a quasifree theory, and we believe that an extension of the methods introduced here will be able to establish localization also at finite temperature, or extended to random disorder.

[1] P. W. Anderson, Phys. Rev. 109, 1492 (1958).

[2] S. Aubry and G. André, Ann. Isr. Phys. Soc. 3, 133 (1980).

[3] G. Roati, C. D’Errico, L. Fallani, M. Fattori, C. Fort, M. Zaccanti, G. Modugno, M. Modugno, and M. Inguscio, Nature (London) 453, 895 (2008).

[4] C. D'Errico, E. Lucioni, L. Tanzi, L. Gori, G. Roux, I. P. McCulloch, T. Giamarchi, M. Inguscio, and G. Modugno, Phys. Rev. Lett. 113, 095301 (2014).

[5] M. Schreiber et al., arXiv:1501.0566.

[6] A. Avila and S. Jitomirskaya, Ann. Math. 170, 303 (2009). 
[7] E. Dinaburg and E. Y. Sinai, Funct. Anal. Appl. 9, 279 (1976).

[8] J. Froehlich, T. Spencer, and T. Wittvwer, Commun. Math. Phys. 88, 151 (1983).

[9] I. Gornyi, A. Mirlin, and A. D. Polyakov, Phys. Rev. Lett. 95, 206603 (2005).

[10] D. M. Basko, I. L. Aleiner, and B. L. Altshuler, Ann. Phys. (Amsterdam) 321, 1126 (2006).

[11] J. Imbrie, arXiv:1403.7837.

[12] V. Oganesyan and D. A. Huse, Phys. Rev. B 75, 155111 (2007).

[13] M. Znidaric, T. Prosen, and P. Prelovsek, Phys. Rev. B 77, 064426 (2008).

[14] A. Pal and D. Huse, Phys. Rev. B 82, 174411 (2010).
[15] V. Ros, M. Mueller, and A. Scardicchio, Nucl. Phys. B891, 420 (2015).

[16] R. Nandkshore and D. Huse, Annu. Rev. Condens. Matter Phys. 6, 15 (2015).

[17] V. Mastropietro, Commun. Math. Phys. 201, 81 (1999); V. Mastropietro, Phys. Rev. B 65, 075113 (2002).

[18] J. Vidal, D. Mouhanna, and T. Giamarchi, Phys. Rev. Lett. 83, 3908 (1999).

[19] S. Iyer, V. Oganesyan, G. Refael, and D. A. Huse, Phys. Rev. B 87, 134202 (2013).

[20] G. Gallavotti, Commun. Math. Phys. 164, 145 (1994).

[21] G. Gentile and V. Mastropietro, Phys. Rep. 352, 273 (2001).

[22] V. Mastropietro, arXiv:1411.0201. 